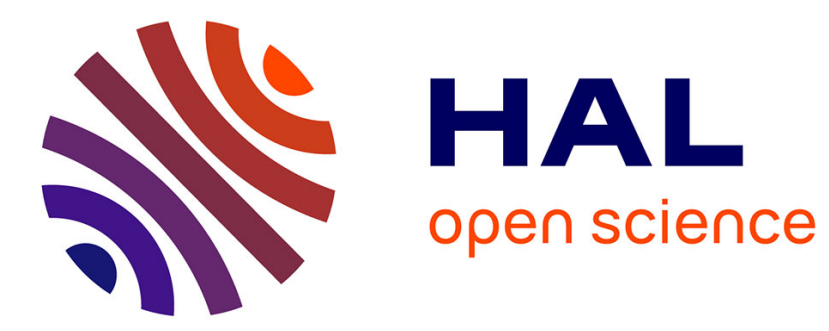

\title{
Deux nouveaux Osiris dans le nord de la Gaule
}

Laurent Bricault

\section{To cite this version:}

Laurent Bricault. Deux nouveaux Osiris dans le nord de la Gaule. Revue du Nord. Histoire \& Archéologie, 1992, 74 (296), pp.179 - 183. 10.3406/rnord.1992.5998 . hal-01817063

\section{HAL Id: hal-01817063 https://hal.science/hal-01817063}

Submitted on 16 Jun 2018

HAL is a multi-disciplinary open access archive for the deposit and dissemination of scientific research documents, whether they are published or not. The documents may come from teaching and research institutions in France or abroad, or from public or private research centers.
L'archive ouverte pluridisciplinaire HAL, est destinée au dépôt et à la diffusion de documents scientifiques de niveau recherche, publiés ou non, émanant des établissements d'enseignement et de recherche français ou étrangers, des laboratoires publics ou privés. 


\section{Deux nouveaux Osiris dans le nord de la Gaule} Laurent Bricault

Citer ce document / Cite this document :

Bricault Laurent. Deux nouveaux Osiris dans le nord de la Gaule. In: Revue du Nord, tome 74, n²96,1992. Archéologie. pp. 179-183;

doi : 10.3406/rnord.1992.5998

http://www.persee.fr/doc/rnord_0035-2624_1992_num_74_296_5998

Document généré le 11/05/2016 
Per toeval werden twee nummievormige Osirisbeelden ontdekt die in 1890 opgegraven werden in de merovingische begraafplaats te Wanquetin (Pas-de-Calais). Men kende daarvoor slechts vijf vondsten in de streek. Een beeldje komt waarschijnlijk uit Beneden-Egypte en kan gedateerd worden in het Late Rijk of in de Ptolemeische periode. Het tweede zou der plaatse kunnen vervaardigd zijn en uit de galloromeinse tijd dateren. Zij maken deel uit van de vele aegyptiaca die in merovingische graven in Norman $\neg$ die en Picardie aangetroffen werden. Zij getuigen van de - nu al goed gedokumenteerde aantrek-kingskracht van de oosterse godsdiensten op de Germaannse volksgroepen die in het Romeinse Rijk binnendrongen. Het magische en beschermende karakter van de Osirisbeelden heeft waarschijnlijk geleid tot hun gebruik als grafgift in de merovingische tijd.

\begin{abstract}
A chance discovery led to the locating of two hitherto unknown nummyform statuettes of Osiris disintered in 1890 from the Merlovingian graveyard of Wanquetin (Pas-de-Calais). They must be added to the five already known statuettes recovered in northern Gaul. One of them probably comes from Lower-Egypt and can be dated back to the Lower-Period or even to Ptolemaic times. The other one might have been made locally and dates back to the Gallo-Roman period. They take place in the already long list of Aegyptiaca recovered in Merlovingian graves in Normandy and in Picardy essentially. In this part of Gaul where oriental cults are now well asserted, they must have translated the attraction exerted by these cults on the Germanic populations entering the Roman world. The magical and protective aspect of the Osiris statuettes would have been then once again at the origin of their burying in the Merlovingian period.
\end{abstract}

\title{
Résumé
}

Une découverte fortuite a permis de dénicher deux nouvelles statuettes d'Osiris momiformes exhumées en 1893 du cimetière mérovingien de Wanquetin (Pas-de-Calais). Elles s'ajoutent aux cinq pièces déjà connues retrouvées dans le nord de la Gaule. Une statuette provient sans doute de BasseEgypte et peut être datée de la Basse-Epoque voire de la période ptolémaïque. La seconde pourrait bien être de facture locale et dater de l'époque gallo-romaine. Elles s'insèrent dans la déjà longue liste des aegyptiaca retrouvés dans des tombes mérovingiennes en Normandie et en Picardie essentiellement. Dans cette région où les cultes orientaux sont désormais bien attestés, elles doivent traduire l'attrait exercé par ceux-ci sur les populations germaniques pénétrant le monde romain. L'aspect magique et protecteur des statuettes osiriennes serait alors une nouvelle fois à l'origine de leur inhumation à l'époque mérovingienne. 


\section{Deux nouveaux Osiris dans le nord de la Gaule}

En 1956, un court article ouvrait la voie à tout un domaine de l'égyptologie, celui de la diffusion des aegyptiaca hors de la vallée du Nil'. Depuis trente ans, les études se sont multipliées. Elles ont trouvé un support de choix dans la remarquable collection des $E P R O^{2}$, fondée par le Pr M.J. Vermaseren. Une méthodique répartition des tâches et des études préside aux destinées de cette série. Le privilège de mettre au point l'inventaire analytique des aegyptiaca exhumés du sol français revient comme il se doit au Pr J. Leclant et à son équipe de l'EPHE. Désireux d'apporter notre modeste contribution à cette grande entreprise, nous voudrions présenter ici deux statuettes que le hasard plaça entre nos mains voici quelques mois ${ }^{3}$.

\section{Les circonstances de la découverte}

Les deux statuettes ${ }^{4}$ d'Osiris momiformes que nous présentons furent retrouvées fortuitement en mars 1988 dans un grenier, à Lécluse (Nord), chez Mme P. Collignon, parmi d'autres petits objets antiques gallo-romains et mérovingiens 5 .

Toutes deux étaient fixées à une planchette de bois et accompagnées d'une étiquette manuscrite en partie effacée. Cette étiquette recense les différentes pièces et indique «Fouilles de Wanquetin 1893 Cimetière mérovingien». Rien ne précise cependant si ces pièces (une douzaine au total) furent découvertes en même temps, ni au même endroit. Tout au plus est-on en droit de penser qu'elles furent inventées dans ce cimetière la même année.

En effet, en 1893, un cimetière mérovingien fut bien mis au jour à Wanquetin (Pas-de-Calais), village situé à une dizaine de kilomètres à l'ouest-sud-ouest d'Arras. Les premières tombes se trouvaient à environ $1,50 \mathrm{~m}$ de profondeur. Aucune fouille d'envergure n'eut lieu cette année-là. Il fallut attendre 1925 pour qu'un érudit local du nom de Cottet procède à des fouilles approfondies du site. 350 tombes recouvertes de grès furent mises au jour, et près de 300 pièces inventoriées. Celles-ci, transportées au Musée Boucher de Perthes d'Abbeville ${ }^{6}$, gagnèrent ensuite Bruxelles après avoir transité par le Musée des Antiquités Nationales de Saint-Germain-en-Laye. Aucun aegyptiacum n'aurait été répertorié dans ce $\operatorname{lot}^{7}$. Une pharmacie trône actuellement sur le site.

1. - J. Leclant, «Notes sur la propagation des cultes et des monuments égyptiens en Occident, à l'époque impériale», $B I F A O S 5$, 1956, p. 173-9, à propos d'un oushebti découvert quatre ans plus tôt à Blendecques (Pas-de-Calais) .

2. - EPRO-Etudes Préliminaires aux Religions Orientales dans l'Empire Romain. Le premier volume est paru en 1961 .

3. - Nous remercions M. et Mme J. Domzalski, actuels propriétaires des statuettes, de nous avoir généreusement permis de les publier.

4. - Sur ce type de statuettes, cf. J. Leclant, "Osiris en Gaule», Studia Aegyptiaca I, 1974, p. 263-85. Cinquante trois Osiris étaient présentés dans cette étude. Nous en connaissons aujourd'hui une soixantaine.

5. - Il s'agit de petites broches, de fibules, de colliers mérovingiens en pierre et en verre, de petites bagues à plaque argentée, d'une amulette en ambre et d'une bague avec chaton gravé sur pierre.

6. - Le Musée Boucher de Perthes, que nous avons contacté, n'a pu nous fournir d'indications complémentaires.

7. - Nous tenons ce renseignement de M. J.L. Podvin, d'après des indications du Musée de Saint-Germain. Qu'ils en soient remerciés. 


\section{Les deux statuettes}

1. Petit Osiris momiforme en bronze

Basse Epoque ou Ptolémaïque

$\mathrm{h}: 12,8 \mathrm{~cm}$

1. max. : $3,1 \mathrm{~cm}$ à hauteur de la coiffe et des coudes

l. min. : $1 \mathrm{~cm}$ à hauteur du cou.

$\mathrm{p}: 123 \mathrm{gr}$

La statuette, représentant Osiris debout, est en bronze plein. D'une seule pièce, elle se termine par un tenon de fixation. Le socle a disparu. Le dos, très plat, indique qu'elle était également plaquée. Le dieu est longiligne, quasi-filiforme. Il porte la couronne atef, avec les deux plumes d'autruche. L'uraeus est très accentué, proéminent ; sa queue est rectiligne. Le visage est étroit et les traits assez grossiers. Le nez est très épaté. La barbe très mince, usée, se fond dans le corps du dieu ; elle se confond presque avec le cou ; une telle barbe est typique de l'Osiris de Busiris défini par Roeder ${ }^{9}$. Les deux mains sont superposées, la droite au-dessus de la gauche ; un espace de $8 \mathrm{~mm}$ sépare les deux mains ; cette position est là aussi caractéristique de l'Osiris de Basse-Egypte ${ }^{10}$; dans la main droite le fouet nekhekh dont le manche repose sur l'épaule droite ; dans la main gauche le sceptre hekat, dont l'extrémité repose sur l'épaule gauche ${ }^{11}$. Les deux attributs du dieu sont usés. Le tenon, prolongeant les jambes à l'équerre du dos, est long de $2 \mathrm{~cm}$. La statuette doit provenir de BasseEgypte.

2. Petit Osiris momiforme en métal cuivreux

Epoque gallo-romaine?

$\mathrm{h}: 11,3 \mathrm{~cm}$; la statuette étant brisée $1 \mathrm{~cm}$ environ au-dessous du bas du sceptre, sa hauteur totale devait avoisiner 15 à $16 \mathrm{~cm}$

1. max. : $4,3 \mathrm{~cm}$ à hauteur des coudes

1. min. : $1,4 \mathrm{~cm}$ à hauteur du cou

p. : 105 gr.

La statuette, en métal cuivreux creux, représente Osiris debout. Le dieu porte la couronne atef. Les deux plumes d'autruche, très stylisées, rectilignes, pourraient indiquer une facture locale. L'uraeus est fort usé ; sa queue est droite. Le visage, aplati, est remarquablement ciselé. La barbe est fine et délicate. Les deux mains sont superposées, la droite au-dessus de la gauche ; elles se touchent ; dans la droite le fouet nekhekh, dont le manche repose sur l'épaule droite; dans la gauche le sceptre hekat dont l'extrémité repose sur l'épaule gauche. 11 incisions zèbrent le manche du fouet, 17 celui du sceptre. Les détails des attributs sont bien visibles. Le sceptre accuse, à hauteur de la main gauche, une courbure singulière. Les mains se détachent du corps, les doigts sont figurés. Les bras, eux, sont quasiment indécelables et se fondent dans le corps.

Comme nous l'avons déjà indiqué, la statuette est brisée à hauteur des jambes. De profil, les plumes d'autruche disparaissent presque du fait de leur minceur extrême. De fait, on imagine plutôt, sous cet angle, une statuette de Ptah, avec son dos caractéristique à hauteur des omoplates ${ }^{12}$. Il pourrait s'agir d'une statuette de facture locale, interprétation de figures osiriennes momiformes certes, mais peut-être également de représentations de Ptah.

8. - Sur les statuettes égyptiennes en bronze, les ouvrages fondamentaux restent :

G. Roeder, Aegyptische Bronzewerke (Pelizaeus-Museum zu Hildesheim, Wissenschaftliche Veröffentlichung, 3), 1937 ; id., Aegyptische Bronzefiguren (Staatliche Museen zu Berlin, Mitteilungen aus der aegyptischen Sammlung, Bd VI), Berlin, 1956. Des Osiris présentent des similitudes avec le nôtre: Bronzewerke $\mathrm{n}^{\circ} 362(\S 92$; pl. $11 \mathrm{~h}, \mathrm{i}), \mathrm{n}^{\circ} 1234(\S 93 ; \mathrm{pl} .11 \mathrm{~d}, \mathrm{e}) ;$ Bronzefiguren $\mathrm{n}^{\circ} 2331$ (§ $\left.185 \mathrm{~g} ; \mathrm{pl} .22 \mathrm{~g}\right)$.

9. - Bronzewerke, p. 240.

10. - Bronzefiguren, § 223b, p. 181-2.

11. - Selon le type le plus courant. Cf. Bronzewerke, $\S 425$ (fouet) et $\S 426 \mathrm{~d}$ (sceptre).

12. - Pour une statuette avec un dos similaire, cf. Bronzefiguren, $\mathrm{n}^{\circ} 8287(\S 185 \mathrm{i}$; pl. 23a,b,c).
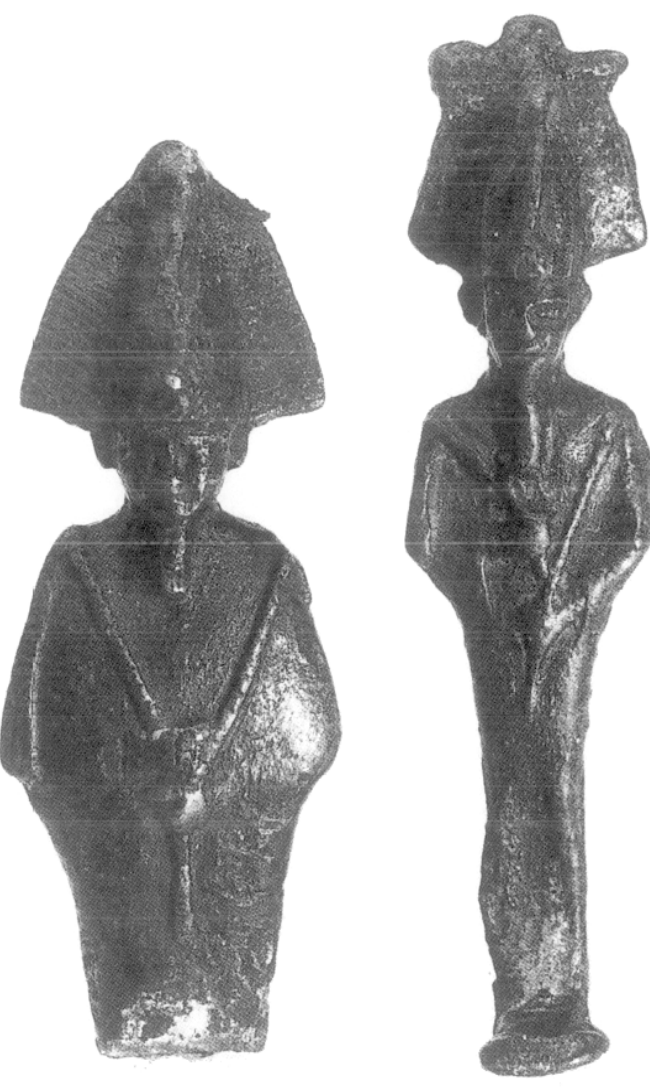

Vus de face

Vus de $3 / 4$ gauche.
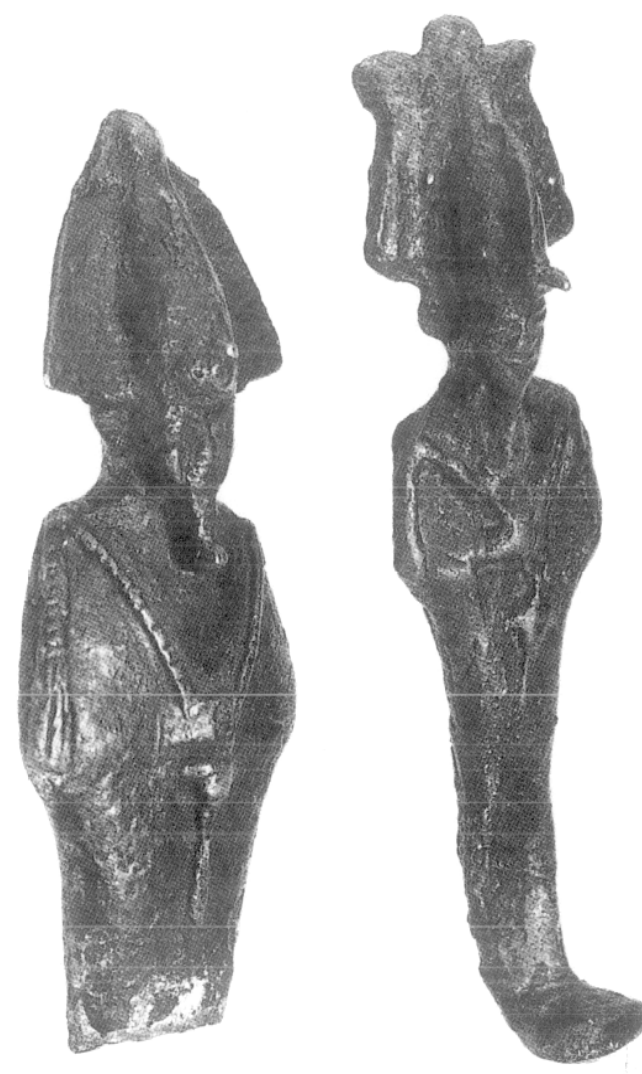

Les deux Osiris de Wanquetin Photo L. Bricault. 


\section{Commentaire}

Trois articles récents viennent de faire le point sur les aegyptiaca du Nord/Pas-de-Calais ${ }^{13}$. Ils mettent en évidence le caractère hypothétique de tout essai de conclusion définitive sur la question, en l'absence de données plus complètes et plus précises sur les objets égyptiens et égyptisants de la région. Tout au plus peut-on constater, pour les Osiris, la relative concentration des trouvailles. L'un fut découvert à Lens, un deuxième à Noyelles-Godault (Pas-de-Calais) ${ }^{14}$, un troisième à Liercourt (Somme) ${ }^{15}$ dans le camp romain et un quatrième au "pied du Mont Saint-Quentin", au nord de Péronne (Somme) ${ }^{16}$. Un cinquième, conservé au Musée des Antiquités Nationales, ayant vraisemblablement appartenu à Boucher de Perthes lui-même, pourrait provenir de la région d'Abbeville ${ }^{17}$.

A cette liste, il convient d'ajouter maintenant les deux statuettes de Wanquetin. Ce qui donne un total de sept Osiris dans un rayon de $45 \mathrm{~km}$ autour de Pas-en-Artois, entre Somme et Pas-de-Calais ${ }^{18}$. Hasard des découvertes, sans doute, mais peut-être pas uniquement.

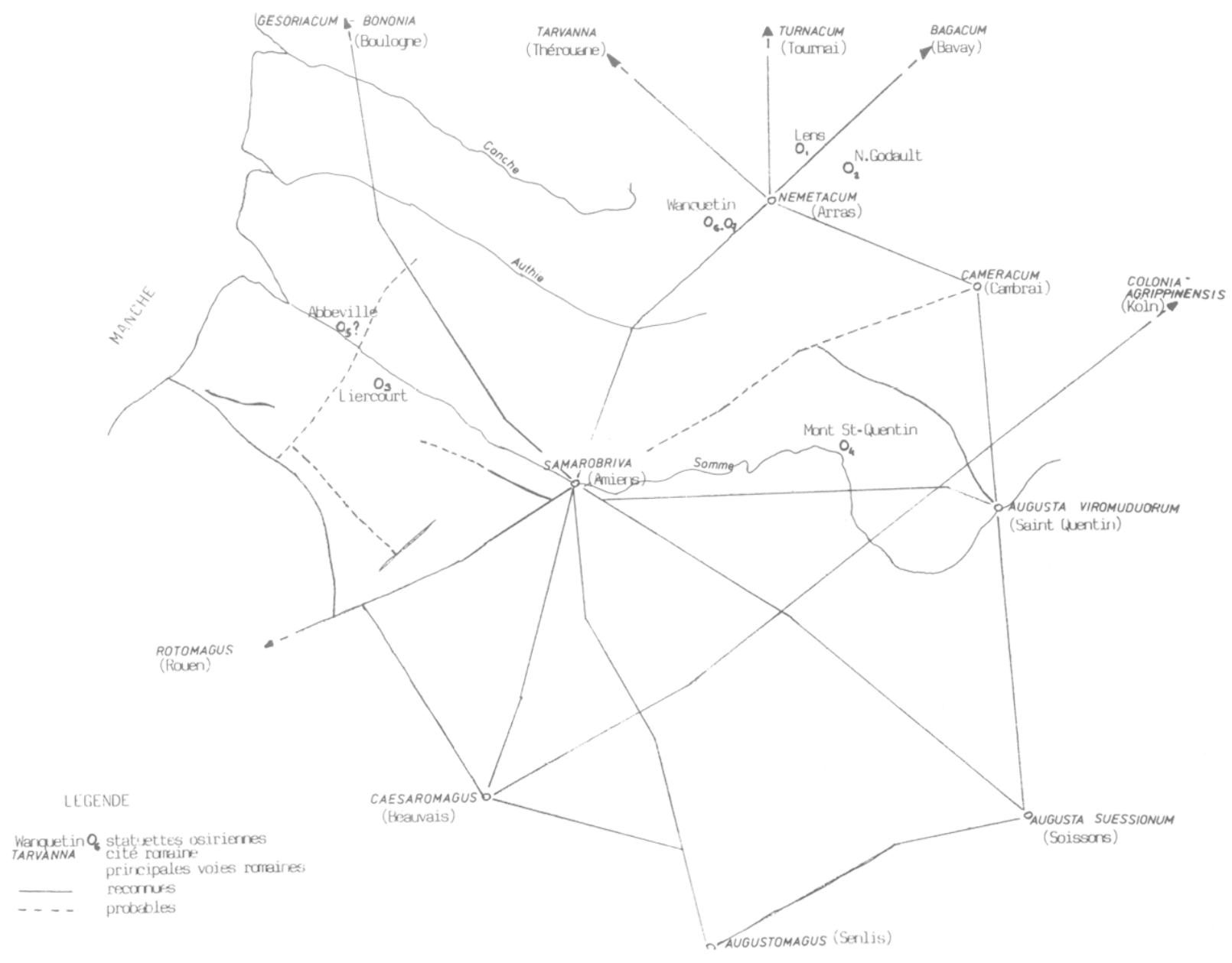

13. - J.-L. Podvin, "Objets égyptiens et égyptisants trouvés dans le Nord et le Pas-de-Calais», Revue du Nord, LXX, 276, 1988, p. 183-90 ; id., "Aegyptiaca du Nord de la Gaule», Bulletin de la Société Egyptologique de Genève 12, 1988, p. 61-70 ; ID., Revue du Nord, Lxxi, 280, 1989, p. 215-220.

14. - Pour de plus amples renseignements sur ces deux statuettes, cf. J. Leclant, «Osiris en Gaule», Studia Aegyptiaca I, 1974, p. 266 et 271 ; J.-L. Podvin, BSEG, p. 63.

15. - Publié dans Les cultes funéraires en Egypte et en Nubie, Catalogue de l'exposition itinérante Calais-Béthune-Dunkerque, Calais 1987, n $^{\circ} 254$, p. 87 ; conservé au Musée Boucher de Perthes d’Abbeville (inv. 1890 ; 67).

16. - Cette pièce, découverte en 1764 et aujourd'hui disparue, a été publiée par J.-L. Podvin, "Aegyptiaca de Picardie», CdE LXV, 129,1990 , p. $43-4$ et carte p. 42.

17. - Conservé sous le no 61445 . Hauteur $9,5 \mathrm{~cm}$. Acéphale. Cette statuette a pu être communiquée à Boucher de Perthes par un habitant du pays. Mais fut-elle exhumée localement? Les renseignements concernant ces deux notes sont de J.-L. Podvin.

18. - Cf. carte. 
On a pu supposer l'existence d'un Iseum à proximité du triangle Lens/Arras/Noyelles-Godault ${ }^{19}$. Rien n'est moins sûr. La découverte récente, à Arras, dans le quartier Baudimont, d'un sanctuaire métroaque peut laisser entrevoir une égyptophilie locale stimulée par ce centre religieux ${ }^{20}$. Ce temple a dû fleurir au III ${ }^{e}$ s. pC. La présence de nombreux soldats ne doit pas y être étrangère.

A Wanquetin même, des érudits locaux font mention d'un camp romain aux Biefs, près du bois de Hauteville, mesurant environ $200 \mathrm{~m}$ sur 100 . Des fragments de tuiles, des briques et des poteries y auraient été trouvés, de même qu'un "sesterce et un adrien du deuxième siècle»" ${ }^{21}$. Ce camp aurait été détruit lors d'une invasion franque. Mais laquelle? Si rien ne prouve encore l'existence de ce camp romain, on peut toutefois avancer que l'antique Wanquetin devait se trouver sur la route qui reliait Arras à Frévent.

Wanquetin fut ensuite occupé par les Mérovingiens, puis les Carolingiens. Quant au cimetière mérovingien proprement dit, il paraît avoir accueilli des sépultures du IVe $^{e}$ au VIII ${ }^{e}$ s. de notre ère, date à laquelle il fut abandonné. Nos statuettes ne seraient certes pas les premiers aegyptiaca à avoir été retrouvés dans des tombes mérovingiennes. Ceci, comme il se doit, concerne tout particulièrement la Picardie et la Normandie.

Pour la Picardie, citons par exemple plusieurs uraei en bronze à pâte cloisonnée ${ }^{22}$, une plume double semblable à celle d'Amon-Rê, en bronze à incrustations de pâte ${ }^{23}$. En ce qui concerne la Normandie, quatre scarabées en fritte bleue furent découverts dans un contexte mérovingien ${ }^{24}$.

On peut vraisemblablement supposer, avec $S$. Aufrère, que ces petits objets, après avoir circulé de main en main, à la suite de trocs, lors des passages de troupes romaines, ont été récupérés par les nouveaux occupants. Ceux-ci les auraient emportés avec eux, soit comme parure funéraire, soit peutêtre même comme amulettes bénéfiques ${ }^{25}$. Il est probable que les deux Osiris de Wanquetin aient répondu à ce désir de protection. Nous pouvons envisager que la statuette égyptienne ait échoué à Wanquetin après maintes tribulations, et que la seconde, égyptisante et de facture locale, soit une œuvre de commande d'un guerrier désireux de posséder lui aussi sa propre statuette-talisman. Il n'est pas douteux que certains chefs barbares aient eu un contact aussi mince, aussi indirect soit-il, avec des Isiaques, fidèles ou membres du clergé. Peut-être même fut-ce simplement par l'intermédiaire de marchands ou de soldats romains du camp de Nemetacum, dans la cité des Atrébates? Quoiqu'il en soit, il est fort probable qu'un, voire deux chefs francs se soient fait enterrer à Wanquetin avec des statuettes osiriennes.

N'oublions pas qu'au milieu du Ive $\mathbf{s}$. pC, un chef alaman, Médérich, ayant passé plusieurs années en Gaule comme otage, semble avoir été initié aux mystères isiaques. En 357, son fils Agénarich porte déjà le nom théophore de Sérapion ${ }^{26}$. Ces chefs comptèrent parmi ceux qu'affronta le futur empereur Julien lors de la bataille de Strasbourg ${ }^{27}$. L'attrait exotique et magique du culte isiaque existait bel et bien pour les Germains.

19. - J. YoyotTe, Sociétés et croyances au temps des pharaons, Catalogue de l'exposition de Boulogne-sur-Mer, 1981, nº 95, p. 13 ; J.-L. PoDvin, BSEG, p. 68 reste lui prudent sur ce point.

20. - C'est dans ce même quartier Baudimont qu'auraient été découverts un certain nombre de sistres en 1793, lors de travaux. Cf. à ce sujet J.-L. Podvin, BSEG, p. 67 et n. 35. Ces pièces ne se trouvent plus aujourd'hui au Musée d'Arras. Un petit singe en terre cuite vient par ailleurs d'être retrouvé lors de la dernière campagne de fouilles. Cf. A. JACQues, Bulletin Municipal de la Ville d'Arras $\mathrm{n}^{\circ} 68,1988$, p. 1. Ce petit singe, datable du IVe $\mathrm{s}$. pC, ne doit sans doute pas être trop rapidement associé aux Isiaques. On a retrouvé en France d'assez nombreuses terres cuites de fabrication gallo-romaine montrant des singes, et ce, en dehors de tout contexte isiaque. Sur les fouilles d'Arras, cf. Archeologia 232, fév. 1988, p. 26 sq.

21. - E. BAYART, Wanquetin à travers les siècles, monographie manuscrite sans date (c. 1984), p. 11. Cf. p. 13-5 sur l'existence d'un cimetière mérovingien à Wanquetin, et les fouilles qui y furent menées.

22. - U. MonNeret de Villard, "Oggetti egizio in una tomba germanica», Aegyptus 3, 1922, p. 315, fig. p. 316 ; J. LeClant, BIFAO 55, 1956, p. 176.

23. - S. DE RICCI, Catalogue of a Collection of Merovingian Antiquities belonging to J. Pierpont-Morgan, Paris 1910; U. MonNeRet de Villard, op. cit. p. 315, fig. p. 316 ; J. LeClant, BIFAO 55, 1956, p. 176.

24. - S. Aufrere, Collections des musées départementaux de Seine-Maritime. Collections égyptiennes, Catalogue d'exposition, Rouen, 1987, nus 414-6, p. 191.

25. - Ibid., p. 215.

26. - J. LeCLANT, BIFAO, 55, 1956, p. 178 ; G. GRIMM, Die Zeugnisse ägyptischer Religion und Kunstelemente im römischen Deutschland, EPRO 12, Leiden, 1969, p. 90 ; L. VIDMAN, Isis und Sarapis bei den Griechen und Römern, Berlin, 1970, p. 156-8 ; R. TuRCAN, "Les religions orientales en Gaule narbonnaise et dans la vallée du Rhône», $A N R W$ II, 18, 1, 1986, p. 480.

27. - Ammien Marcellin xvi, 12, 25. 


\section{Conclusion}

Regrettons de ne pouvoir étayer davantage les hypothèses formulées ici, faute de plus exactes précisions sur les circonstances mêmes de la découverte de ces statuettes, qui viennent toutefois s'ajouter à la déjà longue liste établie voici quinze ans par le Pr. J. Leclant ${ }^{28}$. Souhaitons que de nouvelles fouilles, des inventaires locaux toujours plus approfondis, des pièces enfouies au fond des réserves de certains musées régionaux, des hasards heureux, aussi, nous apportent toujours plus de documentation. Rien de solide ne se bâtit sur des vides.

Laurent BRICAULT

Laurent Bricault, Professeur de Lettres au lycée du Dolmen à Poitiers, doctorant en égyptologie à l'Université de Paris- IV, ParisSorbonne, chercheur associé à l'URA 995 du C.N.R.S., Le Peu, 86130 Saint-Georges-Les-Baillargeaux. 